\title{
Synergetic Strengthening Far Beyond Rule of Mixtures in Gradient Structured Aluminum
}

\section{Rod}

Jordan Moering ${ }^{\mathrm{a}}$, Xiaolong Ma ${ }^{\mathrm{a}}$, Jacob Malkin ${ }^{\mathrm{b}}$, Muxin Yang ${ }^{\mathrm{c}}$, Yuntian Zhu ${ }^{\mathrm{a,d}}$, Suveen

Mathaudhu $^{\mathrm{e}}$

${ }^{a}$ Department of Materials Science and Engineering, North Carolina State University, Raleigh, NC 27695, USA

${ }^{\mathrm{b}}$ North Carolina School of Science and Mathematics, Durham, NC, USA

${ }^{c}$ State Key Laboratory of Nonlinear Mechanics, Institute of Mechanics, Chinese Academy of Sciences, Beijing 100190, PR China;

${ }^{\mathrm{d}}$ School of Materials Science and Engineering, Nanjing University of Science and Technology, Nanjing 210094, People's Republic of China;

${ }^{\mathrm{e}}$ Department of Mechanical Engineering, University of California, Riverside, CA, 92505, USA

Gradient structured metals have been reported to exhibit high strength and high ductility. Here we report that the strength of gradient structured aluminum rod is much higher than the value calculated using the rule of mixtures. The mechanical incompatibility in the gradientstructured round sample produced 3D stress states and extraordinary strengthening and good ductility. An out of plane $\{111\}$ wire texture was developed during the testing, which contributes to the evolution of the stress state and mechanical behavior.

Keywords: Gradient Structure; Strength and Ductility; Surface Hardening; Mechanical Incompatibility; Multiaxial Stress States

(C) 2016. This manuscript version is made available under the Elsevier user license 
Metals exhibiting gradient microstructures have recently become the focus of research for their remarkable ability to produce a superior combination of strength and ductility in metallic systems [1-3]. Gradient structures can be defined as microstructures with macroscopic gradients in one or a combination of the several types of microstructural features including, but not limited to, grain size [1-5], texture [4], dislocation density, twin density [6], precipitates, etc. The most common and well studied gradient structure is grain size gradient structure [1-5], which often consist of nanocrystalline or ultrafine grains at the surface of a tensile sample, which gradually transition to coarse grains in the interior over distances of $\sim 50$ micrometers or longer. Although the means by which gradient microstructures can be created include Surface Mechanical Grinding Treatment, Surface Mechanical Attrition Treatment (SMAT), Ultrasonic Shot Peening [11], Wire Brushing [12], and Air Blast Shot Peening [13], most of the seminal work was undertaken with SMAT $[1,10,12,14-16]$. Much like conventional shot peening, SMAT uses round balls to repeatedly impact the surface of a material. However, the number of impacts and the energy of impacts is often much greater, leading to surface grain refinement to the nanocrystalline regime.

Gradient structures synthesized by SMAT or other approaches are relatively well characterized, but their general effect on global mechanical response is poorly understood. For example, SMAT of IF Steel improved the yield strength by a factor of $\sim 2.5$ while the ductility was only reduced by $\sim 15 \%$ [2]. However, SMAT of pure iron led to similar strength improvements, though ductility was reduced by more than 50\% [15]. In general, yield strength is universally improved in gradient structures, though it seems ductility can either decrease [15], remain more or less unchanged [3], or increase [1]. The discrepancy, in part, must be explained by the mechanism or mechanisms by which gradient structures deform and has been attributed to 
grain growth, dynamic hardening, strain partitioning, and dislocation accumulation at grain boundaries, to name a few $[1,2,16-18]$. One mechanism that has been experimentally verified is the evolution of a multi-axial stress state during tensile testing of the gradient structure [3]. The stress state evolution necessarily rises due to mechanical incompatibility of the hard surface layer and ductile core, and has been observed in flat gradient structures $[2,3]$. The net result of this interaction is that the applied $1 \mathrm{D}$ tensile stress is converted into a $2 \mathrm{D}$ stress state in flat gradient structures during uniaxial tensile deformation. However, in round samples, the ductile core is fully confined by the hard shell and unable to contract in the early tension stage like flat samples due to the lack of freestanding directions. This should lead to a more complex stress state upon loading and could have a greater strengthening effect compared to flat samples [19].

Here we report that gradient structured aluminum rods processed with SMAT have strength that is twice as high as what is predicted by the rule of mixtures, which is dramatically higher than the synergetic strengthening observed in gradient-structured flat samples [3]. To probe the mechanisms behind this surprising observation, the gradient structures of round samples are characterized and the texture evolution was studied with EBSD to explore the stress state evolution during tensile testing.

$1350 \mathrm{Al}$ wire was used for this study and was machined into tensile specimens with a gauge diameter of $5 \mathrm{~mm}$ and a gauge length of $20 \mathrm{~mm}$. The samples were annealed (O tempered) at $370^{\circ} \mathrm{C}$ for 21 hours under vacuum to produce homogenized, coarse grains. This annealing also produced a recrystallized cube texture, which was confirmed with Electron Back Scattered Diffraction (EBSD). The SMAT treatment was conducted using a modified SPEX Mill for 5 minutes using 7 stainless steel balls $6 \mathrm{~mm}$ in diameter. By nature of the randomized impacts, the tensile sample was free to rotate in the vial and exhibited uniform deformation across the gauged 
section. The resulting "as SMAT" sample was cross sectioned and prepared for imaging by conventional polishing techniques and ion milling for 45 minutes. EBSD data was acquired with an Oxford EBSD detector installed in the dual Beam FEI Quanta 3D FEG. Microhardness testing was conducted on the ion polished surface using a Mitutoyo Model HM-11 with a Vickers diamond indenter at a load of $0.001 \mathrm{~N}$. An average of 10 indentations was used for the microhardness profile and Kikuchi patterns from EBSD revealed the prepared sample was free from polishing damage. Figure 1 shows the gradient structure produced by the SMAT surface, the tensile test results, and the resulting hardness profile from surface to interior.

As can be seen in Figure 1, the $\mathrm{O}$ tempered aluminum exhibited a yield strength of 27 MPa which is dramatically increased to $59 \mathrm{MPa}$ after SMAT treatment. Examining the cross section of the as-SMAT sample, it is clear that a deformation gradient, characterized by dislocation accumulation and subgrain boundaries, extends to a depth of $\sim 250 \mu \mathrm{m}$ from the surface. However, the microhardness results show that the hardened layer extends to a depth of $600 \mu \mathrm{m}$ from the surface, and the hardness of the surface layer is only improved by $\sim 50 \%$. In order to surmise the effect of the hardened surface layer, rule of mixtures estimations were calculated using hardness data and the volume fraction of each hardened layer, shown to scale in Fig. 1E. This rule of mixtures analysis has been successfully used in other gradient samples to further reveal the hardening effect of the surface layer $[1,16]$.

There is some debate in the community regarding the relationship between microhardness, yield strength, and ultimate tensile strength. The initial construction by Tabor predicted yield strength in the absence of strain hardening, and found the commonly used empirical relationship, $3 \sigma_{\mathrm{y}}=\mathrm{Hv}$ [20]. When including the effects of strain hardening for soft aluminum alloys, various models have been proposed, but the raw data deviates very little from a 
linear relationship when plotted manually $[21,22]$. In addition, there is also strong experimental evidence corroborating the linear relationship between microhardness and yield strength in 1000 series aluminum [22-27]. Some data show a deviation in the linear relationship between $\sigma_{\mathrm{y}}$ and $\mathrm{Hv}$ in the softened state, but assume a y-intercept of zero, which is not necessarily consistent with experimental results, where y-intercepts of greater than 100 have been reported [28,29]. Therefore, it can generally be assumed that yield strength and microhardness measurements are proportional such that

$$
\sigma_{y}=K H v
$$

where $\mathrm{K}$ is a constant, which may deviate from 3 . Therefore, rule of mixtures predictions can be written as follows:

$$
\frac{\sigma_{g s}}{\sigma_{c g}}=\frac{K \sum V_{f} H_{g s}}{K H_{c g}}=\frac{\sum V_{f} H_{g s}}{H_{c g}}
$$

where $\sigma_{\mathrm{gs}}$ is the yield strength of the gradient structured rod, $\sigma_{\mathrm{cg}}$ is the yield strength of the coarse grained $\mathrm{O}$ tempered rod, $\mathrm{H}_{\mathrm{gs}}$ is the hardness of each layer in the gradient and its respective volume fraction, $\mathrm{V}_{\mathrm{f}}$, and $\mathrm{H}_{\mathrm{cg}}$ is the hardness of the coarse grained $\mathrm{O}$ tempered rod. Examining the data, we see that

$$
\frac{\sigma_{g s}}{\sigma_{c g}}=2.2 ; \quad \frac{\sum V_{f} H_{g s}}{H_{c g}}=1.1
$$

Therefore, there is significant strengthening that is not accounted for by rule of mixtures predictions.

To examine possible strengthening mechanisms at hand, the SMAT-produced microstructure gradient was characterized in cross section using EBSD. Figure 2 shows various 
maps of the aluminum sample and the inverse pole figures of local areas. Typical deformation structures in aluminum can be seen at various depths, including subgrain boundaries, slip bands, and dislocation cells. Although the grain size has not been reduced to the nanocrystalline regime, magnification of the surface in Fig. 1 (e-f) shows the subgrain boundary size approaches $\sim 3 \mu \mathrm{m}$. These subgrain boundaries are typical of the early stages of SMAT processing, and have been observed in samples subjected to small revolutions of High Pressure Torsion (HPT) [10,30]. However, the degree of grain refinement is quite mild at the surface of the SMAT sample which correlates well to the mild improvement in hardness at the surface, as seen in Figure 1.

Beyond the microstructure, there could be other mechanisms that improve the strength of the SMAT sample. It is well known that SMAT samples exhibit high compressive residual stresses, and can develop multiaxial stress states in tension [31,32]. In fact, strengthening from both the initial stress state and the stress state evolution would not be accounted for in the rule of mixture predictions since this model neglects transverse stresses and interaction between layers within the gradient. It is well known that the compressive residual stresses in SMAT structures can exceed the yield strength of the coarse grained core by a factor of 3 and extend to depths of nearly $1 \mathrm{~mm}[31,33]$. In shot peening, similar compressive stresses were shown to increase the yield strength of samples by $\sim 2 \mathrm{x}$ and therefore some of the hardening beyond rule of mixtures can be accounted for by this phenomenon [5]. In some cases, $40 \%$ of the strengthening of gradient structures has been attributed to this residual stress [34]. In addition to this effect, the multiaxial stress evolution, which arises from the mechanical incompatibility between earlyplastic and stable elastic regions, should also contribute to strengthening [2]. At low strains, the stress applied will exceed the yield strength of the coarse grained region, inducing plastic deformation while the surface is still deforming elastically. As the inner region attempts to 
contract, the surface must develop a compressive stress to remain coherent with the shrinking interior, and this phenomenon has been observed experimentally in flat samples [2,3]. In round samples, however, the gradient confines the interior layer, creating 3D internal stresses. Direct observation of the strain accumulation within the gradient is not possible with round samples as with flat samples. Using EBSD, it is possible to get clues about the deformation mechanism of the gradient structure by examining the post-mortem microstructure of the SMAT sample.

In order to investigate the stress state evolution of the gradient layer, samples were taken from the post-mortem "as annealed" and SMAT samples from the uniformly elongated region to compare the microstructure using EBSD. Because the texture symmetry depends on the deformation symmetry, the texture evolution gives insight to the stresses evolved during yielding. If out of plane stresses are operating on the gradient, then the symmetry of these stresses should be evident in the pole figures. After tensile testing, as seen in Figure 3, the postmortem EBSD maps clearly differed, and the $\mathrm{Z}$ and $\theta\{111\}$ and $\{110\}$ axis projection pole figures of the top layer of both samples showed a similar symmetry but the SMAT sample showed out of plane texture symmetry.

In the as-annealed sample, the $\mathrm{Z}$ projection pole figure shows some $\{111\}$ character, and some symmetric "smearing" of the $\{110\}$ about the tensile $(\mathrm{Z})$ axis, all of which is the common texture seen in uniaxial drawing of aluminum [35-37]. Interestingly, the pole figures from the SMAT sample show a similar symmetry, but not around the tensile axis as seen in Fig. 4J. It is clear from the pole figures that the $\theta$ direction develops a similar wire texture to $\mathrm{Z}$ direction of the as annealed sample. This out of plane texture is evidence that a multiaxial stress state evolves during tensile testing that is not present in samples lacking gradient structures. The mechanical mismatch between the hard surface and ductile core causes these multiaxial stresses to develop 
as the gradient is deforming, causing non-uniform deformation and enhanced strengthening while maintaining good ductility.

Despite the evidence of out of multiaxial stress state evolution, it is very difficult to discern the primary strengthening mechanism of this gradient structured aluminum alloy. It was not possible to quantify the residual compression, which has been shown in some cases to greatly affect mechanical performance [5,34]. In addition, the effect of residual stresses on the microhardness testing could skew the hardness due to the compression at the surface and tension in the core. Another factor that could increase the yield strength is the high back stress developed at the plastic-elastic interface of the gradient structure [18]. Also, although the linear relationship between hardness and yield strength was used to predict rule of mixtures calculations in this study, the high rate of strain hardening in aluminum could introduce error into this prediction especially in the measurements of the ductile core and this analysis was used only as an approximation for the gradient structure yield strength. Further studies should explore these confounding effects in more detail to elucidate the strengthening mechanisms in gradient structured aluminum rod. Further, deviations in strength predictions from the rule of mixtures model should be investigated, since this model neglects multiaxial stresses which have been reported here and elsewhere to be non-trivial role in strengthening [3].

Our data demonstrate that aluminum treated by SMAT exhibits a yield strength far beyond what is predicted by rule of mixtures. The gradient structure produced by SMAT produced some improvement in hardness and EBSD maps showed the hardening is accompanied by dislocation accumulation, subgrain boundaries, and very little grain refinement. EBSD of the post-mortem samples showed that the gradient structured aluminum developed an out of plane 
texture that indicates a complex, multiaxial stress evolution during tensile testing that is not present in its coarse grained counterpart.

Unlike previous reports of gradient structures where increased strength and ductility were attributed to grain growth, dislocation accumulation at grain boundaries, and other mechanisms, this report provides evidence that strengthening in round SMAT samples is due to both the complex initial stress state of the material as well as the dynamic strain hardening that occurs within the mismatched layers, which produced 3D complex stress states. These effects lead to synergetic strengthening far beyond of what is predicted by the rule of mixtures in gradient structured rod. Further investigation of the residual stress effect and stress state evolution in gradient structures should shed light on the magnitude of contributions by these strengthening mechanisms.

\section{Acknowledgments}

This work was funded by the US Army Research Office (W911 NF-12-1-0009) the US National Science Foundation (DMT-1104667 and CMMI - 1550986) and the University of California, Riverside. The authors acknowledge the use of the Analytical Instrumentation Facility (AIF) at

North Carolina State University, which is supported by the State of North Carolina and the

National Science Foundation. The authors also acknowledge the constructive discussion with Prof. Wolfgang Pantleon (Technical University of Denmark).

\section{References}

[1] T.H. Fang, W.L. Li, N.R. Tao, K. Lu, Science 331 (2011) 1587-1590.

[2] X.L. Wu, P. Jiang, L. Chen, F. Yuan, Y.T. Zhu, Proc. Natl. Acad. Sci. 111 (2014) 71977201.

[3] X.L. Wu, P. Jiang, L. Chen, J.F. Zhang, F.P. Yuan, Y.T. Zhu, Mater. Res. Lett. 2 (2014) $185-191$. 
[4] J. Moering, X.L. Ma, G.Z. Chen, P.F. Miao, G.Z Li, G. Qian, S. Mathaudhu, Y.T. Zhu, Scr. Mater. 108 (2015) 100-103.

[5] S.P. Wang, Y.J. Li, M. Yao, R.Z Wang, J. Mater. Process. Technol. 73 (1998) 64-73.

[6] Y.J. Wei, Y.Q. Li, L.C. Zhu, Y. Liu, X.Q. Lei, G. Wang, Y.X. Wu, Z.L. Mi, J.B. Liu, H.T. Wang, H.J. Gao, Nat. Commun. 5 (2014).

[7] H.W. Huang, Z.B. Wang, J. Lu, K. Lu, Acta Mater. 87 (2015) 150-160.

[8] Y.S. Zhang, Z. Han, K. Wang, K. Lu, Wear 260 (2006) 942-948.

[9] S. Suresh, Science 292 (2001) 2447-2451.

[10] K. Lu, J. Lu, Mater. Sci. Eng. A 375-377 (2004) 38-45.

[11] Y. Todaka, M. Umemoto, K. Tsuchiya, Mater. Trans. 45 (2004) 376-379.

[12] H. Kitahara, T. Yada, F. Hashiguchi, M. Tsushida, S. Ando, Surf. Coat. Technol. 243 (2014) 28-33.

[13] J.L. Liu, M. Umemoto, Y. Todaka, K. Tsuchiya, J. Mater. Sci. 42 (2007) 7716-7720.

[14] W.Y. Chen, W.P. Tong, C.S. He, X. Zhao, L. Zuo, Mater. Sci. Forum 706-709 (2012) 2663-2667.

[15] H. Murdoch, K. Darling, A. Roberts, L. Kecskes, Metals 5 (2015) 976-985.

[16] X.C. Yang, X.L. Ma, J. Moering, H. Zhou, W. Wang, Y.L. Gong, J.M. Tao, Y.T. Zhu, X.K. Zhu, Mater. Sci. Eng. A 645 (2015) 280-285.

[17] H.W. Höppel, J. May, M. Göken, Adv. Eng. Mater. 6 (2004) 219-222.

[18] X.L. Wu, M.X. Yang, F.P. Yuan, G.L. Wu, Y.J. Wei, X.X. Huang, Y.T. Zhu, Proc. Natl. Acad. Sci. 112 (2015) 14501-14505.

[19] L.J. Ebert, S.S. Hecker, C.H. Hamilton, J. Compos. Mater. 2 (1968) 458-476.

[20] D. Tabor, Rev. Phys. Technol. 1 (1970) 145-179.

[21] S.C. Chang, M.T. Jahn, C.M. Wan, J.Y.M. Lee, T.K. Hsu, J. Mater. Sci. 11 (1976) 623630.

[22] J.R. Cahoon, W.H. Broughton, A.R. Kutzak, Metall. Trans. A 2 (1971) 1979-1983.

[23] N. Rangaraju, T. Raghuram, B.V. Krishna, K.P. Rao, P. Venugopal, Mater. Sci. Eng. A 398 (2005) 246-251.

[24] E.A. El-Danaf, Mater. Sci. Eng. A 487 (2008) 189-200.

[25] Y. Kwon, Scr. Mater. 49 (2003) 785-789.

[26] M. Eizadjou, H.D. Manesh, K. Janghorban, J. Alloys Compd. 474 (2009) 406-415.

[27] E.R. Petty, Metallurgia 63 (1962) 25-26.

[28] P. Zhang, S.X. Li, Z.F. Zhang, Mater. Sci. Eng. A 529 (2011) 62-73.

[29] J.T. Busby, M.C. Hash, G.S. Was, J. Nucl. Mater. 336 (2005) 267-278.

[30] C. Xu, Z. Horita, T.G. Langdon, Mater. Trans. 51 (2010) 2-7.

[31] X. Zhou, F. Belahcene, F. Thomas, J. Lu, in:, 2006.

[32] D.V. Wilson, P.S. Bate, Acta Metall. Mater. 42 (1994) 1099-1111.

[33] T. Roland, D. Retraint, K. Lu, J. Lu, Scr. Mater. 54 (2006) 1949-1954.

[34] J.W. Tian, K. Dai, J.C. Villegas, L. Shaw, P.K. Liaw, D.L. Klarstrom, A.L. Ortiz, Mater. Sci. Eng. A 493 (2008) 176-183.

[35] U.F. Kocks, C.N. Tomé, H.-R. Wenk, eds., Texture and Anisotropy: Preferred Orientations in Polycrystals and Their Effect on Materials Properties, Cambridge Univ. Press, Cambridge, 1998.

[36] T.L. Richard, R.T. Derricott, J. Appl. Crystallogr. 3 (1970) 234-242.

[37] A.B. Lopes, F. Barlat, J.J. Gracio, J.F. Ferreira Duarte, E.F. Rauch, Int. J. Plast. 19 (2003) $1-22$. 


\section{Figure Captions}

Figure 1. (a) EBSD map of the O-tempered aluminum and (b) after SMAT processing. (c) Microhardness profile at various depths and (d) Tensile test results of the SMAT and as-annealed samples with arrow indicating the rule of mixtures prediction of yield strength. (e) Color coded hardness map showing depth of the gradient to scale.

Figure 2. (a) Convention used for axis of the round SMAT sample along the surface normal (R), tensile direction $(Z)$, and radial direction $(\theta)$. (b) Grain size gradient. (c and d) EBSD map of the SMAT sample projected along $\mathrm{Z}$ and grain boundary map, respectively. For the grain boundary maps, red lines indicate misorientation of $>10^{\circ}$ and the black lines indicate misorientations $>2^{\circ}$. (e and f) High resolution EBSD map of the very top surface layer showing numerous subgrain boundaries when projected along $\mathrm{Z}$ and its grain boundary map, respectively.

Figure 3. a) Z projection EBSD map of the uniformly elongated "as annealed" sample after tensile testing and corresponding pole figures showing b) Symmetric smearing of $\{110\}$ about the tensile (Z) axis and c) $\theta$ axis projection. d) Z projection EBSD map of the uniformly elongated SMAT sample after tensile testing and corresponding pole figures projected along e) the $\mathrm{Z}$ axis and $\mathrm{f}$ ) the $\theta$ axis. There is a clear symmetry that develops along the $\theta$ axis in the post mortem SMAT sample not present conventional samples. 

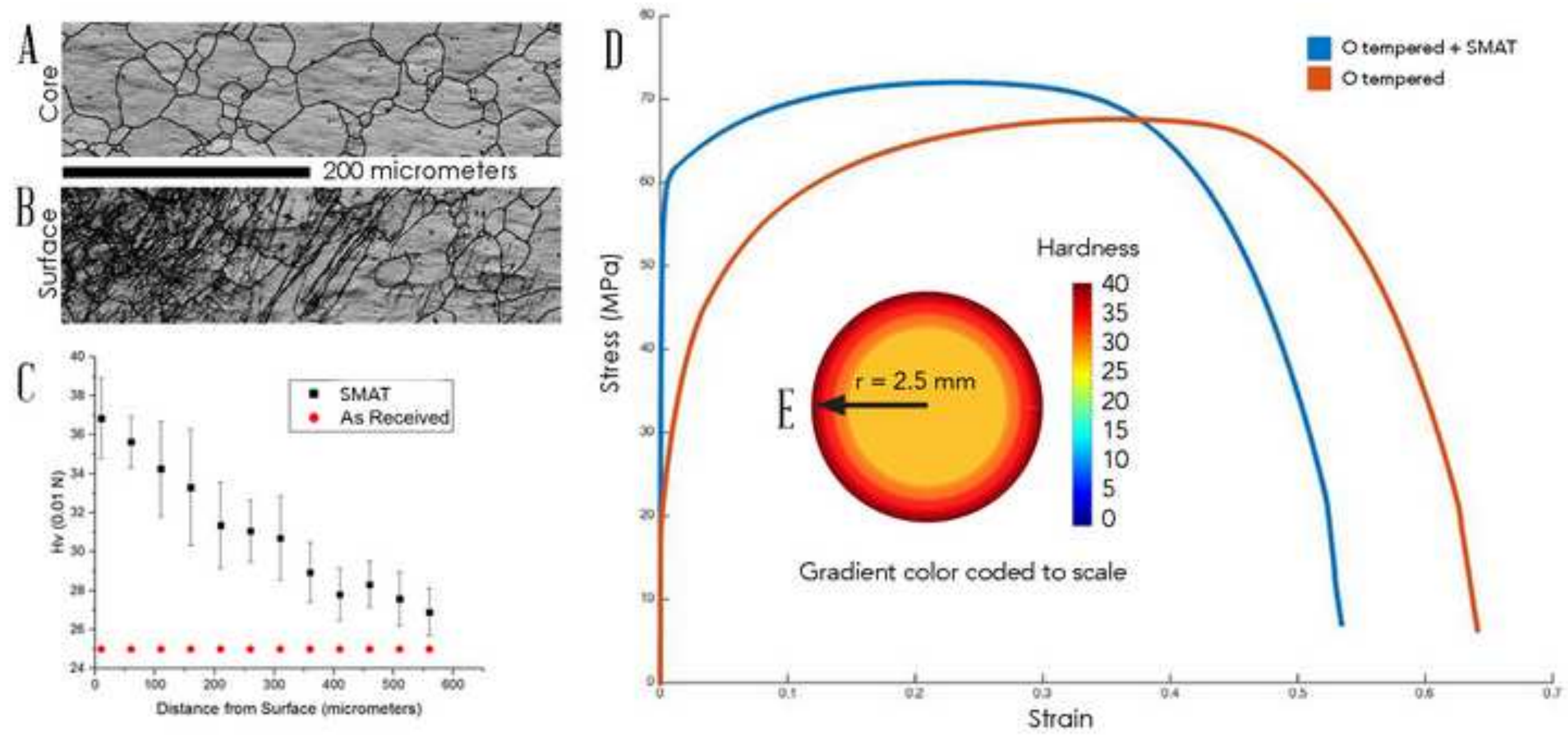

Strain 

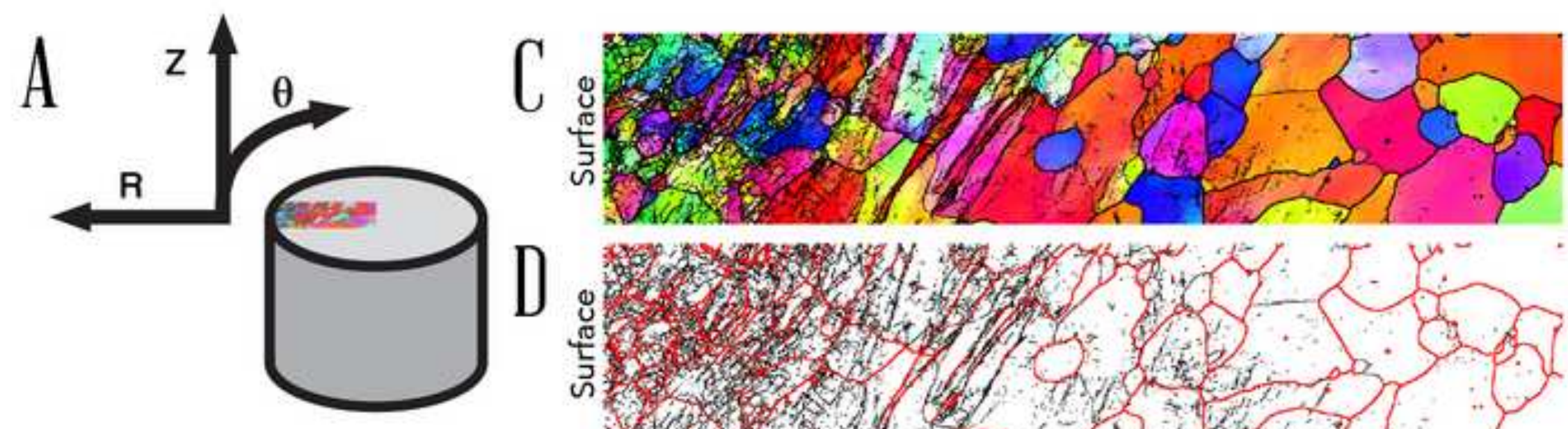

D
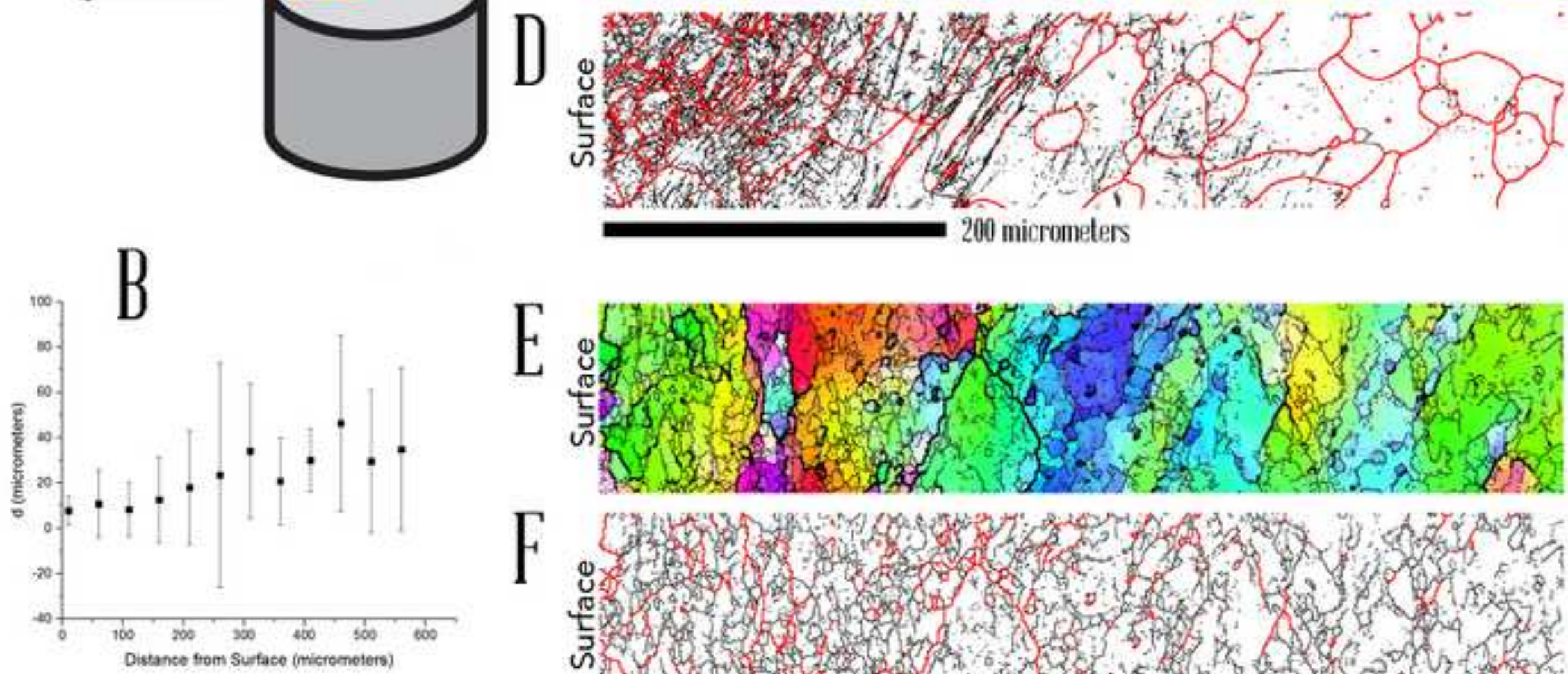

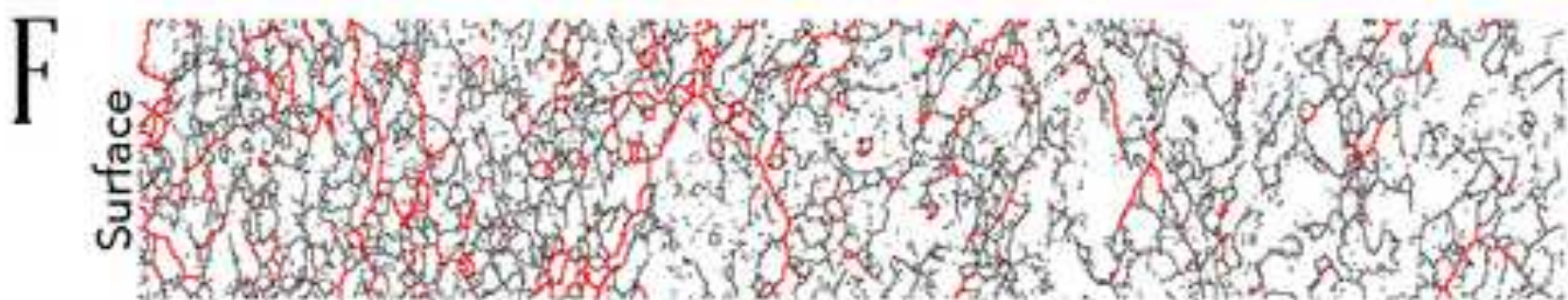
20 micrometers 

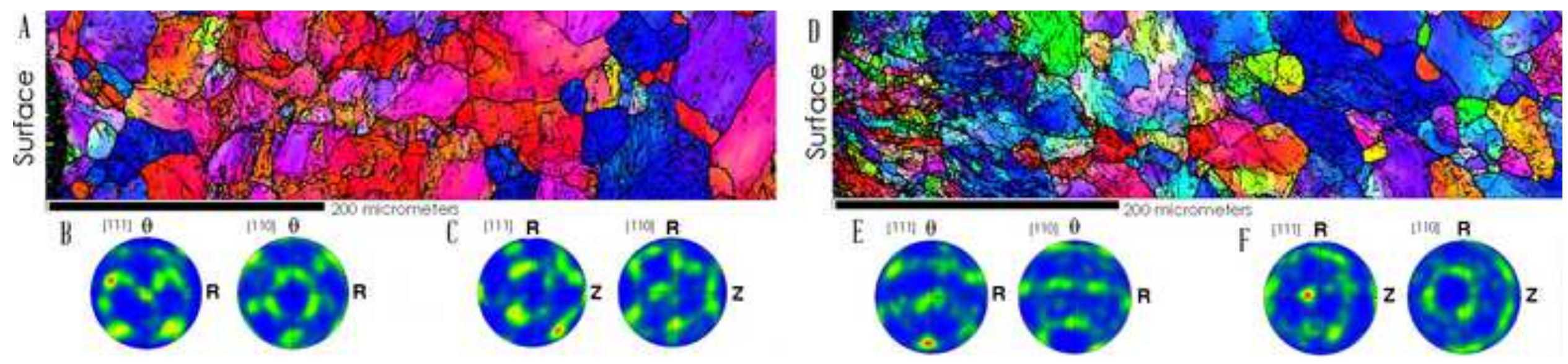

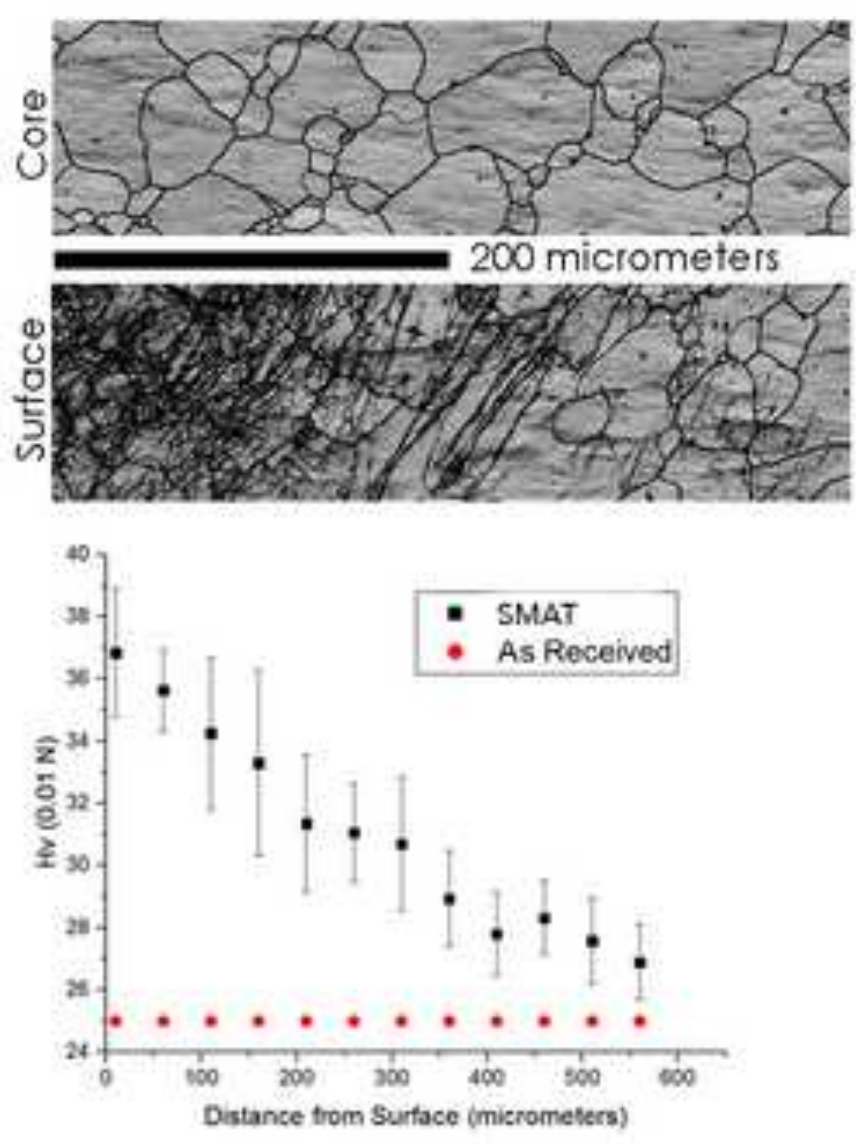

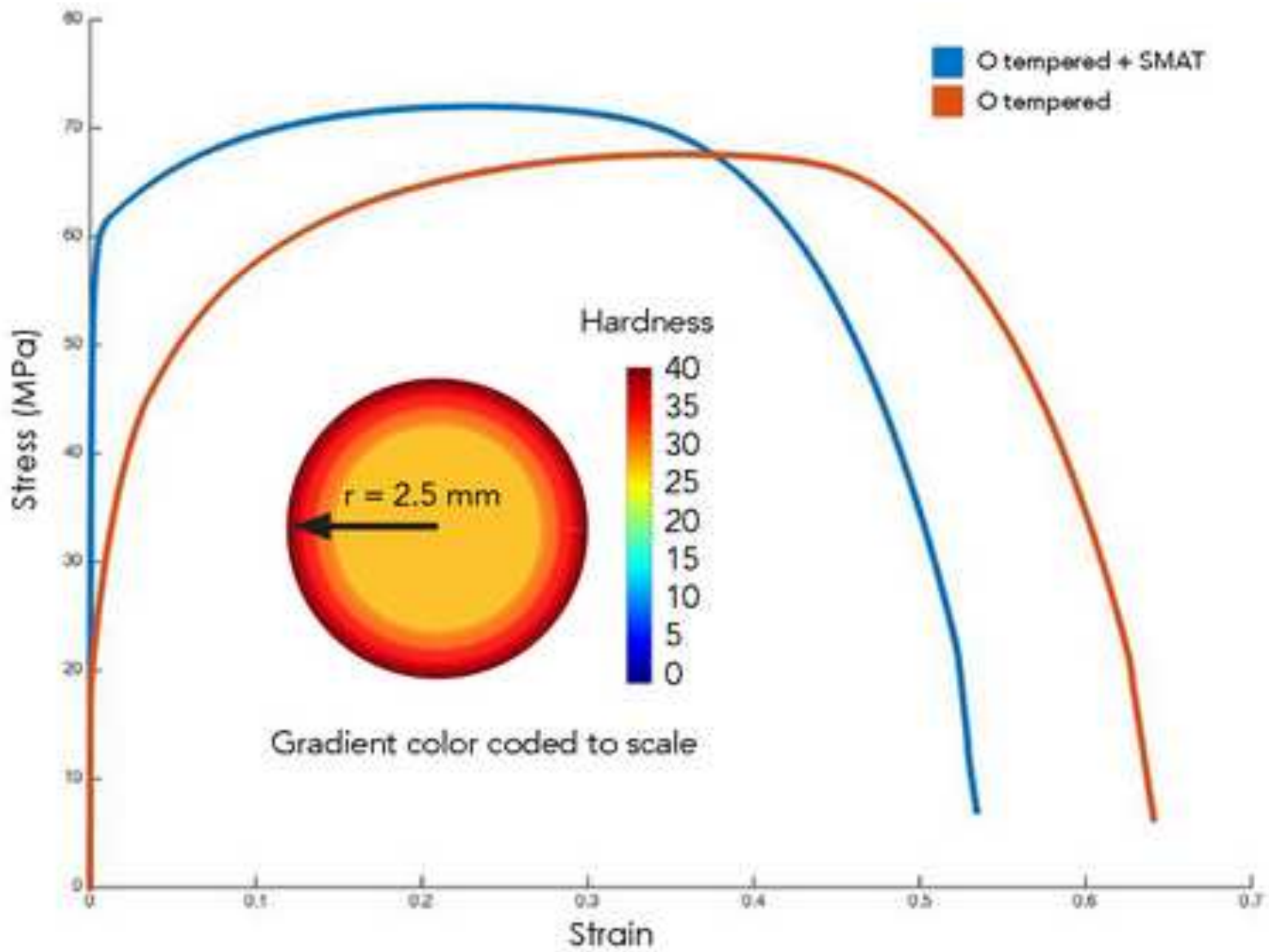

